\title{
Fisioterapia respiratória em crianças com doença falciforme e síndrome torácica aguda
}

\author{
Respiratory therapy in children with sickle cell disease and acute chest syndrome
}

\author{
Sandro Valter Hostyn ${ }^{1}$, Cíntia Johnston², Josefina Aparecida P. Braga ${ }^{3}$, Werther Brunow de Carvalho ${ }^{4}$, Solange Cristiane Nogueira ${ }^{5}$
}

\section{RESUMO}

Objetivo: Realizar uma revisão sistemática da literatura médica para identificar as técnicas de fisioterapia respiratória aplicadas em crianças com doença falciforme e síndrome torácica aguda, bem como descrever seu nível de evidência e recomendação.

Fontes de dados: Revisão bibliográfica nos bancos de dados Medline, Lilacs, SciELO e Cochrane no período de 1995 e 2009, com os descritores: "doença falciforme", "síndrome torácica aguda", "fisioterapia”, "criança", "inspirometria de incentivo", em português e inglês, excluindo-se os estudos de revisão. Os artigos foram classificados por nível de evidência.

Síntese dos dados: Foram encontrados cinco artigos; destes, três utilizaram a inspirometria de incentivo e observaram que ela evita as complicações pulmonares associadas à síndrome torácica aguda (nível de evidência II, II e IV), um deles (evidência II) comparou a inspirometria de incentivo com o dispositivo de pressão expiratória, sem diferenças entre ambos. Um artigo utilizou uma rotina de cuidados, incluindo a inspirometria de incentivo (evidência V), e observou redução do tempo de internação hospitalar e do uso de medicação oral para dor. Outro estudo com a ventilação não invasiva para crianças com desconforto respiratório e com incapacidade de realizar a inspirometria de incentivo relatou melhora da oxigenação e do desconforto respiratório (nível de evidência V).

Conclusões: As técnicas de fisioterapia respiratória com dispositivos de inspirometria de incentivo, de pressão expiratória e a ventilação não invasiva podem ser aplicadas em crianças com doença falciforme e síndrome torácica aguda; o nível de recomendação é C.

Palavras-chave: anemia falciforme; síndrome torácica aguda; criança; fisioterapia/métodos.

\section{ABSTRACT}

Objective: To systematically review the medical literature to identify chest physiotherapy techniques applied to children with sickle cell disease and acute chest syndrome, and to report their level of evidence and recommendation.

Data source: A bibliographic search of published articles found in Medline, Lilacs, SciELO and Cochrane databases, between 1995 and 2009, was carried out using the following keywords: "sickle cell disease", "acute chest syndrome", "physical therapy", "child", "incentive spirometry", in English and Portuguese; all review studies were excluded. The recovered studies were then classified according to their level of evidence and recommendation.
Instituição: Escola Paulista de Medicina da Universidade Federal de São Paulo (Unifesp), São Paulo, SP, Brasil

`Especialista em Fisioterapia Respiratória Pediátrica e Neonatal pelo Instituto da Criança do Hospital das Clínicas da Faculdade de Medicina da Universidade de São Paulo (USP); Supervisor do Curso de Especialização em Fisioterapia Pediátrica e Neonatal da Escola Paulista de Medicina da Unifesp, São Paulo, SP, Brasil

${ }^{2}$ Doutora em Pediatria/Saúde da Criança pela Pontifícia Universidade Católica do Rio Grande do Sul (PUC-RS); Chefe do Serviço de Fisioterapia Pediátrica e Neonatal do Hospital São Paulo da Escola Paulista de Medicina da Unifesp, São Paulo, SP, Brasil

${ }^{3}$ Doutora em Pediatria pela Escola Paulista de Medicina da Unifesp; Professora Adjunta e Chefe do Setor de Hematologia Pediátrica do Departamento de Pediatria da Escola Paulista de Medicina da Unifesp, São Paulo, SP, Brasil

${ }^{4}$ Livre-Docente em Pediatria pela Escola Paulista de Medicina da Unifesp; Professor Titular de Terapia Intensiva e Neonatologia do Departamento de Pediatria da Faculdade de Medicina da USP, São Paulo, SP, Brasil ${ }^{5}$ Especialista em Fisioterapia em Clínica Médica da Escola Paulista de Medicina da Unifesp, São Paulo, SP, Brasil
Endereço para correspondência:

Sandro Valter Hostyn

Rua Itapiru, 572, apto. 76

CEP 04143-010 - São Paulo/SP

E-mail: svhostyn@terra.com.br

Conflito de interesse: nada a declarar

Recebido em: 16/8/2010

Aprovado em: 2/2/2011 
Data synthesis: Five papers were retrieved. Among them, three used incentive spirometry that played an important role in the prevention of pulmonary complications associated with acute chest syndrome (evidence levels II, III and IV); one of these studies (evidence II) compared incentive spirometry versus positive expiratory pressure and did not find differences between them. One paper reported a clinical bundle to improve the quality of care, including incentive spirometry (evidence level V). Incentive spirometry was associated with shorter length of stay and less requirement of oral pain medications. Another study evaluated the effect of non-invasive ventilation on respiratory distress in children that could not perform incentive spirometry and reported improvement in the oxygenation and in the respiratory distress (evidence level V).

Conclusions: Physiotherapy techniques with incentive spirometry device, positive expiratory pressure and non-invasive ventilation can be performed in children with sickle cell disease and acute chest syndrome, with a $\mathrm{C}$ recommendation level.

Key-words: anemia, sickle cell; acute chest syndrome; child; physiotherapy/methods.

\section{Introdução}

A anemia falciforme (AF) é a doença hereditária mais frequente na população brasileira. Tem origem em uma mutação no cromossomo 11, resultando na substituição do ácido glutâmico pela valina na posição 6 da cadeia beta da globulina e, desse modo, na hemoglobina $\mathrm{S}(\mathrm{HbS})$, que irá determinar todas as consequências fisiopatológicas da doença. A doença falciforme inclui os indivíduos com AF, homozigotos para o gene $\mathrm{HbS}$, ou a combinação do $\mathrm{HbS}$ com outros genes que codificam diversas hemoglobinopatias, por exemplo, HbS-C, HbS-talassemia, sendo a AF uma das formas mais grave da doença ${ }^{(1,2)}$.

As manifestações clínicas da doença falciforme podem ser atribuídas às alterações físicas no fluxo sanguíneo em nível da microcirculação ${ }^{(2)}$. Há crises repetidas de agudização, sendo mais frequentes as vaso-oclusivas, que ocasionam, em geral, dor, lesão tecidual e acometimento crônico de diferentes órgãos ${ }^{(1,2)}$. Na DF, o comprometimento pulmonar pode ser de natureza aguda ou crônica. As complicações agudas são representadas pela hiper-reatividade brônquica, pelo tromboembolismo pulmonar e pela síndrome torácica aguda (STA). As complicações crônicas podem levar a alterações da função pulmonar e à hipertensão pulmonar ${ }^{(3-8)}$.

A STA é a segunda causa mais frequente de hospitalização de pacientes com doença falciforme, com taxas altas de morbidade e mortalidade. A STA é definida pela ocorrência de dispneia, dor torácica, febre, tosse e pelo aparecimento de infiltrado pulmonar na radiografia de tórax. Fatores que propiciam a hipoxemia favorecem também a falcização na circulação pulmonar, podendo deflagrar a STA como as infecções, a embolia gordurosa decorrente de infarto da medula óssea, a hiper-hidratação, a sedação excessiva, as atelectasias devido à hipoventilação secundária à dor torácica, o tromboembolismo e o broncoespasmo ${ }^{(3-9)}$.

Os objetivos principais do tratamento da STA são: prevenir as atelectasias e as infecções, promover a oxigenação adequada e evitar a hidratação excessiva ${ }^{(1,2)}$. A fisioterapia respiratória está indicada para melhorar as trocas gasosas e prevenir as comorbidades decorrentes das crises álgicas e vaso-oclusivas $^{(8,9)}$. Nesse contexto, o objetivo deste estudo foi realizar uma revisão sistemática da literatura médica para identificar as intervenções de fisioterapia respiratória passíveis de aplicação em crianças com DF e STA, relatar seu nível de evidência e de recomendação.

\section{Método}

Esta revisão sistemática da literatura foi conduzida nos bancos de dados eletrônicos MedLine, Lilacs, SciELO e Cochrane de acordo com os seguintes critérios de inclusão: artigos publicados no período de 1995 a 2009; estudos realizados com a população pediátrica e submetidos à fisioterapia respiratória. A pesquisa foi realizada nos idiomas português e inglês, respectivamente, com os seguintes descritores: "doença falciforme", "síndrome torácica aguda", "fisioterapia”, "criança", "inspirometria de incentivo", "sickle cell disease","acute chest syndrome", "physiotherapy", "children", "incentive spirometry".

Foram excluídos os estudos de revisão da literatura. Os artigos encontrados foram classificados por nível de evidência e recomendação segundo indicação de Cook et al, publicada em 1992 (Quadro 1) $)^{(10)}$.

\section{Resultados}

Após a revisão dos bancos de dados e aplicados os critérios de exclusão, foram encontrados apenas cinco $\operatorname{artigos}^{(9,11-14)}$ que abordaram o tema desta revisão sistemática. Entre eles, três estudos ${ }^{(9,11,12)}$ utilizaram a inspirometria de incentivo (IS) como técnica de fisioterapia respiratória, em ambiente hospitalar, para as crianças com diagnóstico de DF e STA. Tais investigações mostraram que a IS previne as complicações pulmonares associadas à STA, evitando a hipoventilação, o acúmulo de secreções e as atelectasias. 
Quadro 1 - Classificação das Evidências Científicas

\begin{tabular}{|lr|}
\hline Recomendação & Definição \\
\hline A & Suportadas por pelo menos duas investigações de nível I \\
B & Suportadas por somente uma investigação de nível I \\
C & Suportadas somente por investigações de nível II \\
D & Suportadas por pelo menos uma investigação de nível III \\
E & Suportadas por evidências de nível IV ou V \\
\hline Evidências & Definição \\
\hline Nível I & Estudos aleatórios; grande número de pacientes; resultados claros; risco baixo de erro alfa (falso- \\
& positivos) ou beta (falso-negativos) \\
Nível II & Estudos aleatórios; pequeno número de pacientes; resultados incertos; risco moderado a elevado \\
& de erro alfa (falso-positivo) ou beta (falso-negativo) \\
Nível III & Estudos não aleatórios, controles contemporâneos \\
Nível IV & Estudos não aleatórios, controles históricos e opinião de especialistas \\
Nível V & Séries de casos; estudos não controlados e opinião de especialistas \\
\hline
\end{tabular}

Adaptado de Cook DJ et al,1992 ${ }^{(9)}$.

Um estudo retrospectivo ${ }^{(14)}$ avaliou a implementação de medidas de rotina para os cuidados das crianças hospitalizadas com esse diagnóstico, incluindo a fisioterapia respiratória com IS. Os autores observaram redução do tempo de internação hospitalar e do uso de medicações para dor por via oral. Não houve diferença na frequência de readmissões desses pacientes.

Devido à dificuldade do uso do inspirômetro de incentivo por crianças, Hsu et a ${ }^{(11)}$ compararam a IS com o dispositivo com pressão expiratória positiva (PEP) intermitente em 20 crianças com idade acima de seis anos. Não houve diferença quanto ao conforto e ao tempo de internação hospitalar entre as crianças que utilizaram esses dispositivos. Ambos foram aceitos pelos pacientes e reduziram suas complicações pulmonares.

Ong $^{(12)}$ avaliou 20 crianças hospitalizadas, com idade entre oito e 16 anos, com diagnóstico de DF e dor torácica com o objetivo de avaliar a eficácia da IS na redução da incidência de complicações pulmonares. O estudo indicou que a técnica reduziu as complicações pulmonares entre os pacientes.

No estudo de Padman et $a^{(13)}$, avaliou-se a aplicação da ventilação não invasiva (VNI) no modo ventilatório com dois níveis de pressão (BIPAP), em nove crianças com diagnóstico clínico de DF e STA. Na análise comparativa antes versus após o uso VNI (parâmetros máximos utilizados: pressão inspiratória positiva de $12 \mathrm{cmH}_{2} \mathrm{O}$; pressão positiva expiratória final de $\left.6 \mathrm{cmH}_{2} \mathrm{O}\right)$, observou-se redução da necessidade de oxigênio $(4,1 \pm 3,2$ versus $1,4 \pm 1,7 \mathrm{~L} ; p<0,001)$, melhora da saturação de pulso de oxigênio $(96,3 \pm 2,8 \%$ versus $97,9 \pm 1,6 \% ; p<0,05)$, redução da frequência respiratória $(29 \pm 9$ versus $25 \pm 7 \mathrm{mpm}$; $p<0,05)$ e da frequência cardíaca $(109 \pm 18$ versus $92 \pm 13$ bpm; $p<0,001)$, evitando a progressão da insuficiência respiratória aguda hipoxêmica e a intubação traqueal de $96 \%$ dos pacientes da amostra.

A Tabela 1 apresenta os cinco artigos analisados nesta revisão sistemática, a classificação do nível de evidência e seus resultados.

\section{Discussão}

Os únicos estudos ${ }^{(9,11-14)}$ encontrados que abordam as intervenções de fisioterapia respiratória em crianças com diagnóstico de DF e STA relatam que os dispositivos de IS e aqueles com pressão positiva (PEP ou VNI) são os métodos utilizados com o objetivo de prevenir as complicações pulmonares e, portanto, a progressão da STA, além de atenuar as crises dolorosas.

A inspirometria de incentivo é uma técnica de manutenção da expansão pulmonar, que utiliza fluxo e/ou volume por meio de dispositivos que fornecem um feedback visual ou sonoro. Trata-se de um suporte para manter a expansão pulmonar, simulando o suspiro natural pelo estímulo dado aos pacientes para que realizem inspirações profundas e lentas. A inspirometria de incentivo objetiva educar o paciente, facilitar o controle da respiração, ajudar a visualizar o padrão respiratório e mobilizar volumes pulmonares ${ }^{(15)}$. É indicada na prevenção ou tratamento de atelectasias, na obstrução das vias aéreas, principalmente em pacientes com doença respiratória crônica, alterações pulmonares restritivas, pré e pós-operatório cardíaco e depois de transplantes ${ }^{(15-18)}$.

Não existem contraindicações absolutas descritas na literatura para a aplicação da IS. Entretanto, sua utilização 
instrução e monitoração cuidadosa. Os dispositivos utilizados devem ser de uso individual, assegurando-se as regras de higiene e prevenção de contaminaçãa ${ }^{(15)}$.

Em um estudo randomizado ${ }^{(9)}$, que analisou 38 internações de pacientes com DF e STA, houve diminuição do tempo de internação hospitalar das crianças e adolescentes submetidos à IS durante a fisioterapia respiratória, acompanhada de redução significativa na incidência de complicações pulmonares (pneumonias e atelectasias). Ong ${ }^{(12)}$ também refere que, após a utilização da IS, houve diminuição das complicações pulmonares (hipoventilação e atelectasias) e das crises dolorosas na região torácica, com melhora clínica e dos níveis de saturação de pulso de oxigênio $\left(\mathrm{SpO}_{2}\right)$ dos pacientes analisados. Tais resultados podem ser explicados devido ao uso regular dessa técnica, de baixo custo, capaz de melhorar o desempenho da musculatura respiratória, estimular um padrão respiratório normal e manter a permeabilidade das vias aéreas ${ }^{(19)}$.

A realização da inspirometria de incentivo pode estar limitada pela dor torácica e, na população pediátrica, pela dificuldade de compreensão quanto à técnica. Devido a essa dificuldade, a fisioterapia respiratória com o auxílio do dispositivo de PEP (ou TheraPEP ${ }^{\circledR}$ ou EPAP - pressão positiva expiratória final) é uma opção viável ${ }^{(11)}$.

A respiração com PEP foi desenvolvida com o intuito de favorecer a depuração das secreções das vias aéreas e a aderência ao tratamento, encorajando a independência do paciente e minimizando o desconforto físico. O princípio desse tipo de respiração é semelhante à respiração com lábios pinçados, em que uma resistência é aplicada pela boca durante a expiração, resultando em aumento de pressão na boca, que é transmitida às vias aéreas e contribuir para mantê-las abertas durante a expiração, prevenindo seu fechamento precoce e reduzindo o aprisionamento de ar nos pulmões ${ }^{(15,20,21)}$. Tais efeitos contribuem para promover o movimento da secreção das vias aéreas de médio calibre para as vias aéreas superiores, em direção à boca, facilitando a expectoração.

A pressão positiva expiratória final é produzida pela respiração por meio de uma máscara facial ou de um bucal, provida de uma resistência expiratória (resistor de orifício fixo ou de mola - spring-loaded) e um manômetro de pressão. É possível fornecer ao paciente duas formas de pressão positiva expiratória final: baixo PEP e alto PEP. A de baixo PEP envolve um volume corrente inspiratório e a expiração ativa suave contra a resistência (de mola ou orifício), produzindo pressões positivas expiratórias finais de 10 a $20 \mathrm{cmH}_{2} \mathrm{O}$ no nível boca. A técnica de alto PEP utiliza volumes pulmonares altos e expiração forçada contra uma resistência para gerar pressões expiratórias positivas finais maiores do que $20 \mathrm{cmH}_{2} \mathrm{O}^{(15)}$. O valor dessa resistência deve ser determinado individualmente, de acordo com a capacidade respiratória e o diagnóstico de cada paciente.

A PEP é indicada para reduzir o colapso das vias aéreas e promover a ventilação colateral, sendo aplicada na asma e na doença pulmonar obstrutiva crônica para reduzir o aprisionamento de ar, assim como na fibrose cística e na bronquite crônica para mobilizar secreções ${ }^{(20,21)}$. A técnica é efetiva para prevenir ou reverter atelectasias. Os mesmos efeitos fisiológicos da PEP podem ser benéficos para otimizar a demanda de medicação por aerossol, especialmente de broncodilatadores. Os volumes aumentados e tempos expiratórios mais longos podem melhorar a distribuição e a deposição da medicação na via aérea ${ }^{(15)}$.

A PEP apresenta a vantagem da autoadministração, ser bem-aceita pelos pacientes por seu fácil manuseio, baixo custo e efeito rápido, sendo mais efetiva em casos de média a moderada obstrução de vias aéreas. As reações adversas podem estar associadas às técnicas de depuração das vias aéreas em pacientes com obstrução grave, devido à imposição de um trabalho respiratório adicional, que pode levar ao risco de fadiga da musculatura respiratória e queda da saturação de pulso de oxigênio ${ }^{(15)}$.

Quanto à aplicação da VNI, no modo ventilatório BIPAP, os dados encontrados sugerem que esse pode ser aplicado para melhorar a oxigenação, diminuir o trabalho respiratório em pacientes com STA, prevenir a progressão para insuficiência ventilatória aguda e sua aplicação precoce pode evitar admissões na unidade de cuidados intensivos, reduzindo assim, os custos da internação ${ }^{(13,22-25)}$.

De qualquer modo, deve-se citar que esta revisão sistemática apresenta algumas limitações, dentre as quais destaca-se a publicação escassa sobre o tema e a qualidade metodológica dos estudos analisados (dois deles ${ }^{(9,11)}$ randomizados, mas com pequeno número de pacientes, um deles ${ }^{(12)}$ não controlado e outros dois ${ }^{(13,14)}$ retrospectivos), sugerindo a necessidade de novos estudos com métodos mais apurados e maior número de pacientes.

Pode-se concluir que esta revisão sistemática da literatura identificou que as intervenções de fisioterapia respiratória por meio do uso dos dispositivos de IS, PEP e VNI podem ser aplicadas em crianças com DF e STA, com nível de recomendação C. 


\section{Referências bibliográficas}

1. Brasil - Agência Nacional de Vigilância Sanitária (Anvisa). Manual de diagnóstico e tratamento de doenças falciformes. Brasília: Anvisa; 2002.

2. Campanaro CM, Lyra IM, Viana MB, Araújo PI, Figueira CM, Anjos AC et al. Doença falciforme. In: Braga JA, Tone LG, Loggetto SR, editores. Hematologia para o pediatra. São Paulo: Atheneu, 2007. p. 73-89.

3. Moreira GA. Repercussões respiratórias da anemia falciforme. J Bras Pneumol 2007;33:18-20.

4. Souza LC, Viegas CA. Qualidade de sono e função pulmonar em adolescentes portadores de anemia falciforme clinicamente estáveis. J Bras Pneumol 2007;3:275-81.

5. Martins WA, Mesquita ET, Cunha DM, Ferrari AH, Pinheiro LA, Romeo LJ et al. Alterações cardiovasculares na anemia falciforme. Arq Bras Cardiol 1998;70:365-70.

6. Frei-Jones MJ, Baxter AL, Rogers ZR, Buchanan GR. Vaso-occlusive episodes in older children with sickle cell disease: emergency department management and pain assessment. J Pediatr 2008;152:281-5.

7. Gualandro SF, Fonseca GH, Gualandro DM. Complicações cardiopulmonares das doenças falciformes. Rev Bras Hematol Hemoter 2007;29:291-8.

8. Vichinsky EP, Neumayr LD, Earles AN, Williams R, Lennete ET, Dean D et al. Causes and outcomes of the acute chest syndrome in sickle cell disease. National acute chest syndrome study group. N Engl J Med 2000;342:1855-65.

9. Belett PS, Kalinyak KA, Shukla R, Gelfrand MJ, Ucknagel DL. Incentive spirometry to prevent acute pulmonary complications in sickle cell diseases. N Engl J Med 1995;333:699-703.

10. Cook DJ, Guyatt GH, Laupacis A, Sackett DL. Rules of evidence and clinical recommendations on the use of antithrombotic agents. Chest 1992;102 (Suppl 4):305S-11.

11. Hsu LL, Batts BK, Rau JL. Positive expiratory pressure device acceptance by hospitalized children with sickle cell disease is comparable to incentive spirometry. Respir Care 2005; 50:624-7.

12. Ong GL. Incentive spirometry for children with sickle cell disorder. Nurs Times 2005;101:55-7.

13. Padman R, Henry M. The use of bilevel positive airway pressure for the treatment of acute chest syndrome of sickle cell disease. Del Med J 2004;76:199-203.
14. Co JP, Johnson KB, Duggan AK, Casella JF, Wilson M. Does a clinica pathway improve the quality of care for sickle cell anemia? Jt Comm J Qual Saf 2003;29:181-90.

15. Stopiglia MC, Coppo MR, Carvalho FL. Dispositivos auxiliares de fisioterapia respiratória. In: Barbosa AP, Johnston C, Carvalho WB, editores. Fisioterapia. Série Terapia Intensiva Pediátrica e Neonatal. $5^{\text {th }}$ ed. São Paulo: Atheneu; 2009. p. 79-114.

16. Barth J. Recommandations des journées internationales de kinésithérapie respiratoire instrumentale (JIKRI). Cah Kinésithér 2001;209:11-25.

17. Patterson JE, Bradley JM, Hewitt O, Bradbury I, Elborn JS. Airway clearance in bronchiectasis: a randomized crossover trial of active cycle of breathing techniques versus Acapella. Respiration 2005;72:239-42.

18. Weindler J, Kiefer RT. The efficacy of postoperative incentive spirometry is influenced by the device-specific imposed work of breathing. Chest 2001;119:1858-64.

19. Hristara-Papadopoulou A, Tsanakas J, Diomou G, Papadopoulou O. Current devices of respiratory physiotherapy. Hippokratia 2008;12:211-20.

20. Placidi G, Cornacchia M, Polese G, Zanolla L, Assael BM, Braggion C Chest physiotherapy with positive airway pressure: a pilot study of short-term effects on sputum clearance in patients with cystic fibrosis and severe airway obstruction. Respir Care 2006;51:1145-53.

21. Denehy $L$, Berney $S$. The use of positive pressure devices by physiotherapists Eur Respir J 2001;17:821-9.

22. Carvalho WB, Horigoshi NK. Conceitos básicos e contra indicações da VNIPP. In: Barbosa AP, Johnston C, Carvalho WB, editores. Ventilação não invasiva em neonatologia e pediatria. São Paulo: Atheneu; 2007. p. 1-16.

23. Essouri S, Chevret L, Durand P, Haas V, Fauroux B, Devictor D. Noninvasive positive pressure ventilation: five years of experience in a pediatric intensive care unit. Pediatr Crit Care Med 2006;7:329-34.

24. Joshi G, Tobias JD. A five-year experience with the use of BiPAP in a pediatric intensive care unit population. J Intensive Care Med 2007;22:38-43.

25. Yañez LJ, Yunge M, Emilfork M, Lapadula M, Alcántara A, Fernández C et al. A prospective, randomized, controlled trial of noninvasive ventilation in pediatric acute respiratory failure. Pediatr Crit Care Med 2008;9:484-9. 